\title{
Implementation of one-dimensional quantum walks on spin-orbital angular momentum space of photons
}

Pei Zhang, 迥 Bi-Heng Liu, ${ }^{2}$ Rui-Feng Liu, ${ }^{1}$ Hong-Rong Li, ${ }^{1}$ Fu-Li Li, ${ }^{1}$ and Guang-Can Guo ${ }^{2}$

${ }^{1}$ MOE Key Laboratory for Nonequilibrium Synthesis and Modulation of Condensed Matter, Department of Applied Physics, Xi'an Jiaotong University, Xi'an 710049, People's Republic of China

${ }^{2}$ Key Laboratory of Quantum Information, University of Science and

Technology of China, CAS, Hefei 230026, People's Republic of China

\begin{abstract}
Photons can carry spin angular momentum (SAM) and orbital angular momentum (OAM), which can be used to realize a qubit system and a high-dimension system respectively. This spin-orbital system is very suitable for implementing one-dimensional discrete-time quantum random walks. We propose a simple scheme of quantum walks on the spin-orbital angular momentum space of photons, where photons walk on the infinity OAM space controlled by their SAM. By employing the recent invention of an optical device, the so-called 'q-plate', our scheme is more simple and efficient than others because there is no Mach-Zehnder interferometer in the scheme.
\end{abstract}

PACS numbers: 03.67.-a, 03.67.Lx, 42.50.Xa

a zhang.pei@stu.xjtu.edu.cn 


\section{INTRODUCTION}

Classical random walks (CRW) play an important role in classical algorithms and have many applications in many realms of science [1]. While in the quantum worlds, quantum random walks (QRW) are markedly different due to the phenomenon of interference [2], which might lead to extensive applications in quantum information science. The recent presentation of several quantum algorithms based on QRW has shown the exponential speed up over classical algorithms [3] as the most prominent algorithms suggested by Shor [4] and Grover [5]. Most recently, Childs has shown that, in principle, any quantum algorithm can be recast as a QRW algorithm [6]. Meanwhile, numerous schemes of QRW have been proposed by using various systems, including ion traps [7], microwave or optical cavities [8], nuclear magnetic resonance (NMR) system [9], optical lattices [10], linear optical elements [11 14], quantum accelerator modes [15] and superconducting circuit quantum electrodynamics [16]. All these results are advancing forward towards the ultimate goals of quantum computation.

CRW can take many different forms. For simplicity, we consider the discrete time random walk on a line. It can be understood as a "walker" standing at the starting point $(x=0)$, holding a coin, decides which side (right or left) he should take his one step toward by flipping the coin (suppose with head to right and tail to left). He flips the coin, walks one step to right or left based on the flipping results. After repeating this process $n$ times, he will randomly walk to a position $x$. This is a simple model of CRW on a line. We can get the probability $P_{n}(x)$ of the "walker" being found at $x$ after $n$ steps:

$$
P_{n}(x)=\frac{1}{2^{n}}\left(\begin{array}{c}
n \\
\frac{x+n}{2}
\end{array}\right) .
$$

From Eq. 1, we can easily calculate that the mean value is zero and the standard deviation of this distribution is $\sigma_{c}=\sqrt{n}$, which we will find being distinctly different to QRW later. QRW occurs on quantum particles, where their motion described by wave-function and the "coin" changed into "quantum coin" [17]. Under this version, "quantum walker" does not walk one step determinately to one position. So there must be interference between wave-function at different positions. Suppose we define the two level states as $|\downarrow\rangle$ and $|\uparrow\rangle$, which correspond to the two sides of "quantum coin", and one dimensional discrete position $|x\rangle$ ( $x$ is integer) as the walk space. Then we can perform a Hadamard translation $(\hat{H})$ to the "quantum coin" as a "quantum coin" tossing. The direction of "quantum walker" walks to every one step is decided by the results of tossing "quantum coin", for $|\downarrow\rangle$ steps to $|x-1\rangle$ and $|\uparrow\rangle$ steps to $|x+1\rangle$. The operator of QRW can be written as:

$$
\hat{U}=e^{i \hat{p} \hat{\sigma}_{z}} \hat{H} .
$$

Where $\hat{p}$ is the pseudo-momentum operator of the particle confined to one dimension, and $\hat{\sigma}_{z}$ is the pauli- $z$ operator acting on the qubit [7]. Then the state of the system after $n$ steps is

$$
\left|\Psi_{n}\right\rangle=\left(e^{i \hat{p} \hat{\sigma}_{z}} \hat{H}\right)^{n}\left|\Psi_{i n}\right\rangle
$$

For different initial states of "quantum coin", the results of QRW are very different. We can also calculate the mean value which is nonzero and the standard deviation of the probability distributions $\sigma_{q} \approx \frac{\sqrt{2}}{2} t[18]$. These are two main different features between CRW and QRW.

Photons are well known to be extremely powerful for carrying and manipulating quantum information. QRW have been experimentally demonstrated in linear optical systems [13, 14]. Ref. [13] realized a QRW by employing photon's polarizations and space modes. But the number of linear optical elements grows quickly when steps increasing and the results are not very perfect. Ref. [14] carried out an experiment of QRW by using photon's up-down routes and orbital angular momentum (OAM). This setup is simpler than [13] because there are only two space modes and the photons "walk" on OAM space. However, these two experiments both have a critical shortcoming, that is, they both need Mach-Zehnder interferometers. The instability of interferometer directly leads the precision decreasing when steps of random walk increasing. In this paper, we design an experimental scheme of QRW by using linear optical system. We employ photon's spin angular momentum (SAM) as "quantum coin" and OAM space as one-dimensional walk space. By using q-plates [19], our scheme is very simple and efficient for there is no Mach-Zehnder interferometer in the setup. This paper is organized as follow: first we briefly introduce the OAM of photons and the q-plates; then we will give a detailed description and discussion of our experimental scheme; last is the conclusion.

\section{ORBITAL ANGULAR MOMENTUM AND Q-PLATE}

As we know, light beams have polarizations and helical modes. For photons, it is corresponding to spin angular momentum (SAM) and orbital angular momentum (OAM), respectively. SAM is associated with the circular polar- 
ization of light, each photon carries $\pm \hbar$ angular momentum depending on the handedness of the polarization. OAM is associated with transverse amplitude and phase profile in the cross section orthogonal to the propagation axis, and one photon carries an intrinsic orbital angular momentum given by $m \hbar$ if the wave function contains phase term $\exp (i m \phi)$ [20]. Since the quantum nature of OAM was demonstrated by Mair et al. [21], more and more studies have focused on encoding the high-dimensional quantum information by using OAM of a single photon [22]. These works open up a new possibility for generation and manipulation of higher-dimensional quantum states and implementation of higher-dimensional quantum information processing protocols, by using OAM as a quantum information carrier.

When light propagates through a medium, the SAM and OAM are not always simultaneous independent coupling with the matter [23]. In vacuum or in a homogeneous and isotropic medium, the SAM and OAM are separately conserved. But in a medium which is inhomogeneous and anisotropic, they are not. In appropriate condition, the exchange with matter of the SAM affects the direction of the exchange of the OAM, and it is possible that the angular momentum only converting between SAM and OAM. A device called q-plate is used to conduct this function. Q-plate is a slab of liquid crystal (or uniaxial birefringent medium) with special structure, and it is essentially a retardation wave plate whose optical axis is aligned nonhomogeneously in the transverse plane in order to create a topological charge $q$ in its orientation. If we assume $q=1$, for example, each photon being converted from right circular to left circular change its spin z-component angular momentum from $-\hbar$ to $+\hbar$, and the orbital z-component angular momentum changes $-2 \hbar$. Therefore, the total variation of angular momentum is nil, and there is no net transfer of angular momentum to the q plate. The plate acts only as a medium for the conversion between spin and orbital angular momentum. If $q \neq 1$, it will exchange an angular momentum of $\pm 2 \hbar(q-1)$ with each photon. Using Dirac marks, suppose the initial state is $\left|\uparrow_{S}, m_{L}\right\rangle$ or $\left|\downarrow_{S}, m_{L}\right\rangle$, which refers to the photon carrying SAM with $\hbar$ (left circular polarization) or $-\hbar$ (right circular polarization) and OAM with $m \hbar$. The action of a tuned q-plate of charge $q=1$ on this state can be summarized as follows:

$$
\left(\left|\uparrow_{S}, m_{L}\right\rangle,\left|\downarrow_{S}, m_{L}\right\rangle\right) \stackrel{q-\text { plate }}{\longrightarrow}\left(\left|\downarrow_{S}(m+2)_{L}\right\rangle,\left|\uparrow_{S},(m-2)_{L}\right\rangle\right)
$$

In this way, a photon in an OAM state $m=0$ is transformed into $m= \pm 2$, or a photon in $m= \pm 2$ to $m= \pm 4$ or $m=0$, depending on the polarization. This spin-controlled-orbital transform is very suitable for implementing quantum walks. Here we use the character of infinity space of OAM and the properties of q-plates to plan out an implementation of one-dimensional discrete time QRW, where SAM refer to the quantum "coin" and $m$ of OAM refer to the photon "positions" when random walk. We will give a detailed explanation later.

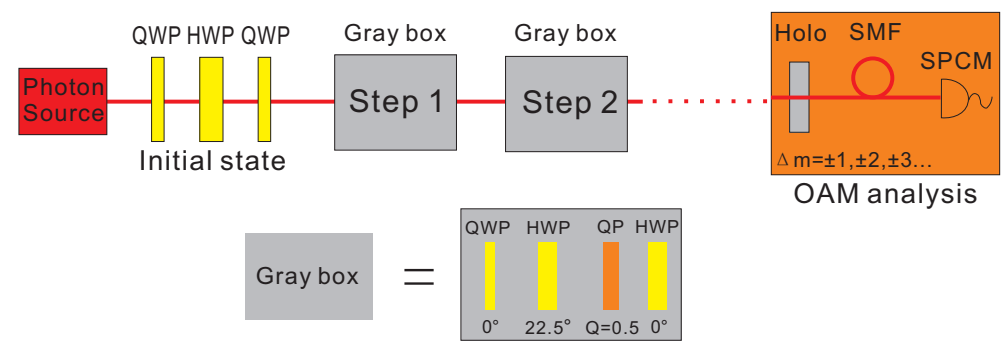

FIG. 1. Experimental scheme of one-dimensional quantum walks on spin-orbital angular momentum space of photons. One half wave plate (HWP) sandwiched by two quarter wave plates (HWP) can realize any single bit unitary operation, which is used as a initial state preparation. Gray box, which is made up by two HWPs, one QWP and one q-plate (QP), performs the QRW operation $\hat{U}$. An n-steps QRW can be achieved by applying $n$ times of gray box. At end of the setup is the OAM analysis system, which contains a computer-generated hologram (Holo), a single mode fiber (SMF) and a single photon counting module (SPCM). When we change different holograms (with different $\Delta m$ ), we can get the probabilities of the photons being in each OAM state.

\section{EXPERIMENTAL SCHEME}

QRW on the OAM space of photons have been studied both theoretically [12] and experimentally [14]. However, the steps of QRW can hardly be increased in the experiment, because of the stability of Mach-Zehnder interferometer decreasing quickly when the arms of interferometer increasing. We plan out a QRW scheme without interferometers which can achieve a multi-step QRW stably and can be easily implemented in the lab. In our scheme, we can use the q-plates with $q=0.5$ [24] where the OAM index $m$ changes to $m \pm 1$ when passing it.

Our experimental scheme is shown in Fig. 1. The single photon source (can be realized by deep attenuating the coherent laser or by nonlinear process) emits one photon with linear polarization (suppose horizontal polarization) and 
OAM $m=0$. The state can be writen as $|H, 0\rangle=|(R+L) / \sqrt{2}, 0\rangle$, where $\mathrm{H}$ is short for horizontal polarization, $\mathrm{R}$ and $\mathrm{L}$ are short for right circle polarization and left circle polarization. We can also use a set of wave plates made by one half wave plate (HWP) sandwiched by two quarter wave plates (QWP) [25], to prepare different initial polarization states as quantum coin states if needed. Then we will toss the quantum coin and decide which direction should step to. This process is achieved by the gray box shown in Fig. 1. Hadamard gate $(\hat{H})$ is a kind of quantum coin tossing, which can realize translation as following:

$$
\begin{aligned}
& \hat{H}|R\rangle=\frac{1}{\sqrt{2}}(|R\rangle+|L\rangle) \\
& \hat{H}|L\rangle=\frac{1}{\sqrt{2}}(|R\rangle-|L\rangle) .
\end{aligned}
$$

In the gray box, a QWP at $0^{\circ}$ and a HWP at $22.5^{\circ}$ make up a $\hat{H}$ gate for $|R\rangle$ and $|L\rangle$ states. After flipping the coin, then the photon will step to $m+1$ when coin state is $|R\rangle$ or to $m-1$ when coin state is $|L\rangle$. Here the photon's QRW positions are denoted by the OAM numbers of photon. From Eq. (4), we know a q-plate can easily realize this process combining with a HWP at $0^{\circ}$ for spin reverse. So, the QRW operator $\hat{U}$ is achieved by the gray box. Then we can apply this operation $n$ times as a $n$ steps QRW. If the initial state is $\left|\Psi_{i n}\right\rangle=|R, 0\rangle$, we can deduce three steps for example:

$$
\begin{aligned}
&\left|\Psi_{i n}\right\rangle \stackrel{\hat{U}}{\longrightarrow} \frac{1}{\sqrt{2}}(|R,-1\rangle+|L, 1\rangle) \\
& \quad \stackrel{\hat{U}}{\longrightarrow} \frac{1}{2}(|R,-2\rangle+|R, 0\rangle+|L, 0\rangle-|L, 2\rangle) \\
& \quad \stackrel{\hat{U}}{\longrightarrow} \frac{1}{2 \sqrt{2}}(|R,-3\rangle+|L,-1\rangle+2|R,-1\rangle-|R, 1\rangle+|L, 3\rangle) .
\end{aligned}
$$

From the third step, we can see the distribution of probabilities of photons being found at different positions (photon's OAM) is asymmetric, which is very different to CRW. At the end of this setup, we should detect the probabilities of the photons being in a OAM state $|m\rangle$. This is achieved by using different computer-generated holograms (Holo) 26] with $\Delta m= \pm 1, \pm 2, \cdots, \pm n$ and a single mode fiber (SMF). We know that the single mode fiber can only pass $T E M_{00}$ mode light and has a strong absorption to other higher order modes. So, to detect photon in OAM state $|m\rangle$, we can use a hologram of $\Delta m=-m$ to change the photon into OAM state $|0\rangle$, which then can pass through the SMF and detected by a single photon count modulator (SPCM).

The efficiency of our setup is very high due to high transmitted efficiency of q-plate [19] and linear optical elements. We know that the different OAM photons will spatially separate in their radial pattern by free propagation. This may induce imperfection when take a long free propagation. However, this can be resolved by placing the q-plates in the common focal plane of two lens (the lens are not appeared in our setup) 27]. It is might be inconvenient in changing the different holograms for position detecting. For $n$ steps QRW, the detection needs to change $2 n$ different holograms. But this is not very hard to our scheme, because it can keep a long time stable for all the different OAM modes are in one spatial mode and no use of Mach-Zehnder interferometer.

There is another work of experimental realization of QRW by taking advantage of OAM of photons as walk space (see Ref. 14]). Comparing with the scheme of Ref. [14], our scheme has two main improvements: the first one is the efficiency, because the transmitted efficiency of q-plate is higher than computer-generated holograms; the second is the stability, our scheme is inherently stable for no need of Mach-Zehnder interferometer, because we know that Mach-Zehnder interferometer is very sensitive with environment especially for long arms. So, by employing SAM as quantum coin and using q-plate, as we analyzed above, the scheme of this paper is more efficient and simple. QRW on a line can be understood as an interference phenomenon [2], although there is no Mach-Zehnder interferometer in our scheme, there still exists interference. From Eq. (6), we can see that in the third step of QRW, the spin interference between state $|R, 0\rangle$ and $|L, 0\rangle$ occurs on the third Hadamard gate.

\section{CONCLUSION}

By employing photons SAM and OAM, we put forward a simple and efficient scheme of discrete-time QRW on a line. We have introduced an optical device, the gray box, by which the QRW operation $\hat{U}$ can be realized. By applying $n$ times of gray box, it is very simple to realize a $n$ steps QRW. The efficiency of our scheme is high and the stability is very good, which are suitable for implementing multi-step QRW. We think our scheme is very simple to do some QRW algorithm experimentally. 


\section{ACKNOWLEDGEMENT}

The authors thank Professor L. Marrucci for helpful discussion on q-plates, and acknowledge the National Fundamental Research Program (2010CB923102), National Natural Science Foundation of China (Grant No. 60778021, 10674106 and 10774117) for supporting this work.

[1] M.N. Barber and B.W. Ninham, Random and Restricted Walks: Theory and Applications (Gordon and Breach, New York, 1970); R. A. Dana and M. Jeanblanc, Financial Markets in Continuous Time (Springer, Berlin, 2002).

[2] P. L. Knight, E. Roldán and J. E. Sipe, Phys. Rev. A 68, 020301(R) (2003).

[3] A. M. Childs, R. Cleve, E. Deotto, E. Farhi, S. Gutmann, D. A. Spielman, Proc. 35th ACM Symposium on Theory of Computing (STOC 2003), pp. 59-68; N. Shenvi, J. Kempe, K. BirgittaWhaley, Phys. Rev. A, 67, 052307 (2003).

[4] P. W. Shor, in Proceedings of the 35th Annual Symposium on Foundations of Computer Science (IEEE Computer Society Press, Los Alamitos, CA, 1994), p. 124.

[5] L. K. Grover, Phys. Rev. Lett. 79, 325 (1997).

[6] A. M. Childs, Phys. Rev. Lett. 102, 180501 (2009).

[7] B. C. Travaglione and G. J. Milburn, Phys. Rev. A 65, 032310 (2002); H. Schmitz, R. Matjeschk, Ch. Schneider, J. Glueckert, M. Enderlein, T. Huber, and T. Schaetz, Phys. Rev. Lett. 103, 090504 (2009); P. Xue, B. C. Sanders, and D. Leibfried, Phys. Rev. Lett. 103, 183602 (2009).

[8] B. C. Sanders, S. D. Bartlett, B. Tregenna, and P. L. Knight, Phys. Rev. A 67, 042305 (2003); P. L. Knight, E. Roldan, and J. E. Sipe, Opt. Commun. 227, 147 (2003); T. Di, M. Hillery, and M. S. Zubairy, Phys. Rev. A 70, 0323042004.

[9] J.-F. Du, H. Li, X. Xu, M. Shi, J. Wu, X. Zhou, and R. Han, Phys. Rev. A 67, 042316 (2003); C. A. Ryan, M. Laforest, J. C. Boileau, and R. Laflamme, Phys. Rev. A 72, 062317 (2005).

[10] W. Dür, R. Raussendorf, V. M. Kendon, and H.-J. Briegel, Phys. Rev. A 66, 052319 (2002); M. Karski, L. Föster, J.-M. Choi, A. Steffen, W. Alt, D.r Meschede, and A. Widera, Science 325, 5937 (2009).

[11] H. Jeong, M. Paternostro and M. S. Kim, Phys. Rev. A 69, 012310 (2004);

[12] X.-B. Zou, Y.-L. Dong and G.-C. Guo, New J. of Phys. 8, 81 (2006).

[13] B. Do, M. L. Stohler, S. Balasubramanian, C. Eash, E. Fischbach, M. A. Fischbach, A. Mills and B. Zwickl, J. Opt. Soc. Am. B 22, 499-504 (2005).

[14] P. Zhang, X.-F. Ren, X.-B. Zou, B.-H. Liu, Y.-F. Huang and G.-C. Guo, Phys. Rev. A 75, 052301 (2007).

[15] Z.-Y. Ma, K. Burnett, M. B. d'Arcy and S. A. Gardiner, Phys. Rev. A 73, 013401 (2006).

[16] P. Xue, B. C. Sanders, A. Blais, and K Lalumière, Phys. Rev. A 78, 042334 (2008).

[17] J. Kempe, Cont. Phys. 50, 339 (2003).

[18] H. A. Carteret, M. E. H. Ismail and B. Richmond, J. Phys. A 36, 8775 (2003).

[19] L. Marrucci, C. Manzo, and D. Paparo, Phys. Rev. Lett. 96, 163905 (2006); L. Marrucci, C. Manzo, and D. Paparo, Appl. Phys. Lett. 88, 221102 (2006).

[20] L. Allen, M. W. Beijersbergen, R. J. C. Spreeuw, J. P. Woerdman, Phys. Rev. A 45, 8185 (1992).

[21] A. Mair, A. Vaziri, G. Weihs and A. Zeilinger, Nature 412, 313 (2001).

[22] S. Franke-Arnold, L. Allen, and Miles. Padgett, Laser \& Photon. Rev. 2, 299 (2008).

[23] H. He, M. E. J. Friese, N. R. Heckenberg, and H. Rubinsztein-Dunlop, Phys. Rev. Lett. 75, 826 (1995);N. B. Simpson, K. Dholakia, L. Allen, and M. J. Padgett, Opt. Lett. 22, 52 (1997); B. Piccirillo and E. Santamato, Phys. Rev. E 69, 056613 (2004).

[24] Generally, q-plate with $q=1$ is easy to manufacture and widely used in the former articles. Here we can also use $q=1$, which can realize a QRW on a even $m$ OAM space.

[25] B. G. Englert, C. Kurtsiefer and H. Weinfurter, Phy. Rev. A 63, 032303 (2001).

[26] V. Y. Bazhenov, M.V. Vasnetsov, and M. S. Soskin, JETP Lett. 52, 429 (1990); N. R. Heckenberg, R. McDuff, C. P. Smith, and A. G. White, Opt. Lett. 17, 221 (1992).

[27] S. Slussarenko, E. Karimi, B. Piccirillo, L. Marrucci, and E. Santamato, Phys. Rev. A 80, 022326 (2009). 